\title{
ELECTROMYOGRAPHY SIGNAL ON BICEPS MUSCLE IN TIME DOMAIN ANALYSIS
}

\author{
Abu Bakar Yahya ${ }^{1 *}$, Wan Mohd Bukhari Wan Daud ${ }^{1}$, Chong Shin Horng ${ }^{1}$ and \\ Rubita Sudirman ${ }^{2}$ \\ ${ }^{1}$ Faculty of Electrical Engineering, Universiti Teknikal Malaysia Melaka, \\ Hang Tuah Jaya, 76100 Durian Tunggal, Melaka, Malaysia \\ *Email: abubakar1110@gmail.com \\ Phone: +6065552237; Fax: +6065552222 \\ ${ }^{2}$ Faculty of Electrical Engineering, Universiti Teknologi Malaysia, \\ 81310 Skudai, Johor, Malaysia
}

\begin{abstract}
Features extraction is important for electromyography (EMG) signal analysis. The paper's objective is to evaluate the features extraction of the EMG signal. The experimental set-up for EMG signal acquisition followed the procedures recommended by Europe's Surface Electromyography for Non-invasive Assessment of Muscle (SENIAM) project. The EMG signal's data were analysed in the time domain to get the features. Four features were considered based on the analysis, which are IEMG, MAV, VAR and RMS. The average muscle force condition can be estimated by correlation between the EMG voltage amplitude with linear estimation with the full-wave rectification method. The R-squared value determined the correlation between the EMG voltage amplitude with the loads. IEMG was chosen as the reference feature for estimation of the muscle's force due to its R-squared value equal to 0.997. By referring to the IEMG, the linear equation obtained from the correlation was used for estimation of the muscle's force. These findings can be integrated to design a muscle force model based on the biceps muscle.
\end{abstract}

Keywords: Electromyography (EMG); time domain; force estimation; non-invasive.

\section{INTRODUCTION}

Electromyography (EMG) is a one-dimensional time series signal of the electrical muscle activity that reflects the physiology of the neuromuscular system upon a certain excitation. The technique of detecting, processing and analysing EMG signals is known as electromyography. It is becoming one of the most important physiological signals now being widely studied and used in clinical and engineering applications. However, the intention of using these signals in the field of information technology is still new. This signal is commonly a function of time and is definable in terms of its amplitude, frequency and phase $[1,2]$. EMG is a complex signal affected by many aspects such as physiological and anatomical properties and characteristics of the instrumentation. It is different from one person to another. Understanding the EMG signals involves understanding the skeletal muscles and the methods used to generate the biosignals. It also involves consideration of the specific mechanisms and phenomena that affect the signals [3]. The EMG signals are helpful in real-time monitoring. They include realtime information about the electrical activity of a particular muscle which is related to 
the muscle's force. The correlation between EMG signals and muscle force has been reviewed during isometric contractions and dynamic contractions. Many methods and approaches to modelling for computing the muscle's force have been suggested, but these models could not be validated due to the lack of an accurate and efficient experimental procedure to compute the muscle's force [4-6]. Recently, most muscle coordination is reviewed from the surface EMG activity.

Non-invasive measurement is one of the techniques that have been used in computing the surface muscle's activity. It is important due to its ability to support many critical biomedical applications for monitoring, diagnostics and therapies [7]. Surface EMG (sEMG) is a non-invasive measurement, which means a procedure that does not involve tools that break the skin or physically enter the body. In other words, sEMG is a result derived in space and time of electrical activities in muscles under the skin. The applications of sEMG signals are included in rehabilitation and assistive technology. The most important application of the sEMG signal in these fields is to control a prosthesis or other assistive equipment by applying different patterns of sEMG signals. Furthermore, the EMG signal is a sequence of voltages recorded from a contracting muscle over time. The potentials are recorded in the voltage field generated by active muscle cells or fibres of a contracting muscle [8]. The amplitude of the sEMG signal depends on the muscle type and condition during the observation process and it is in a range of about $\mu \mathrm{V}$ to $\mathrm{mV}$.

There are two main types of electrodes that are used to measure and record the EMG signals: needle and surface electrodes. The selection of electrode depends on the purpose and application of the experiment to be carried out. For clinical usage, indwelling electrodes such as concentric needle, monopolar needle and single-fibre needle electrodes are used to measure the EMG signals. Needle electrodes are inserted directly into a muscle and are able to measure EMG activity in deep muscles. However, this may cause some pain when inserted into the muscle. Needle electrodes can be difficult to position suitably within a muscle and may move during muscle contraction. Generally, signals measured via needle electrodes are known as intramuscular EMG signals or more specifically known as needle EMG signals [8-11]. Meanwhile, surface electrodes are used in non-invasive application. They are applied to the skin overlying a muscle. The use of surface electrodes is recommended by the Surface Electromyography for the Non-Invasive Assessment of Muscles (SENIAM) project. The SENIAM project is a European concerted action in the Biomedical Health and Research Program (BIOMED II) of the European Union [12]. They are convenient and easy to use and do not cause pain. Signals that are measured via the surface electrodes are known as surface EMG (sEMG) signals. The sEMG signals do not provide much information about the deep muscle activity. This is because of the filtering characteristics of the conductive properties of the overlying muscles and other subcutaneous tissues, even though they are easy to detect. The sEMG signals are helpful for diagnostic purposes because they can be used to get detailed temporal and spatial information about the fibres of a motor unit [8].

This paper aims to evaluate features extracted from sEMG signals on the biceps brachii muscle during specific exercises in lifting the arm. This research describes the signal processing for single channel sEMG signals on the biceps brachii muscle. The sEMG signals are then analysed and their amplitude is estimated with the muscle's force. 


\section{METHODS AND MATERIALS}

\section{Experimental Procedures}

In a pre-experiment, the subject must undergo skin preparation procedures. If the skin preparation is done properly, the skin typically gets a light red colour that indicates a good skin impedance condition. After skin preparation, the skin impedance is then measured. The Ohm-resistance between the electrode pair is measured to verify the skin impedance. This experiment is to consider the skin impedance. Table 1 shows the skin impedance reference value of the Ohm-resistance measurement. The quality of an EMG measurement strongly depends on proper skin preparation and electrode positioning. The aim of skin preparation is to get a stable electrode contact and low skin impedance. Selection of the electrodes depends on the practical condition and specific aim. In this paper, the non-invasive measurement was used for acquiring the sEMG signal. Surface electrodes are used to measure the EMG of a superficial large muscle. They are noninvasive, easy and convenient to use in a laboratory environment. The EMG signal originally initiates from the action potentials of motor units and passes through muscle, skin tissues, electrode-skin interface, amplifier and recorder. These specific mediums and stages act as different filters that change the amplitude and frequency of the original signal from the motor units.

Table 1. Skin impedance reference value for the Ohm-resistance measurement.

\begin{tabular}{ll}
\hline Impedance range $(\mathrm{kOhm})$ & \multicolumn{1}{c}{ Recommendation } \\
\hline $1-5$ & Very good condition \\
$5-10$ & Good and recommended if feasible \\
$10-30$ & Acceptable for easy condition \\
$30-50$ & Less good, attention is needed \\
$>50$ & Should be avoided or requires a second cleaning run \\
\hline
\end{tabular}

Figure 1 shows the overall work of the proposed study. The EMG signals were recorded using three electrodes: two electrodes for recording the EMG signal and one electrode as a reference electrode. The electrodes were placed on the right forearm biceps brachii muscles of a healthy subject. The placement of the sEMG electrodes on the skin is according to SENIAM. This experiment was done in a quiet room to minimize the noise and get better signals $[7,13]$. The subject was standing up straight to get a straight and upright posture, as shown in Figure 1(a). Data for this experiment were recorded continuously. These data were to be used for estimating the average muscle force at the biceps brachii muscle. The subject was required to lift his hand freely without load and then with loads of $2 \mathrm{~kg}, 4 \mathrm{~kg}$ and $6 \mathrm{~kg}$. The repeatability for each load is 30 times. Thus, the subject was supervised by an instructor to lift his hand without load and with a dumbbell load of $2 \mathrm{~kg}, 4 \mathrm{~kg}$ and $6 \mathrm{~kg}$. Six $\mathrm{kg}$ is considered the maximum load that a normal and healthy person, male or female, can lift using a dumbbell with a single hand. A person with a dominant right hand was chosen for this experiment. This was in order to minimize interference by signals from the heartbeat and electrocardiogram (ECG). During the experiment, the right hand of the subject was lifted from $\theta_{\mathrm{E}}=0^{\circ}$ to $\theta_{\mathrm{E}}=145^{\circ}$ measured at the elbow joint, where $145^{\circ}$ is the maximum range of flexion. The subject lifted his hand with the specified weights, starting from no load to the heavier weights. The subject lifted the load from $\theta_{\mathrm{E}}=0^{\circ}$ in 
2 seconds and held it at $\theta_{\mathrm{E}}=145^{\circ}$ for 6 seconds; then lowered back the load to $\theta_{\mathrm{E}}=0^{\circ}$ in 2 seconds, as shown in Figure 2. Holding the load for a period of 6 seconds ensures that the EMG signals are reaching their maximum amplitude. During the experiment, the subject should not move his shoulders but only lift the load by using his hand. Figure 1(b) shows the position of the hand during flexion. The subject had 5 minutes of rest when changing from one weight of load to another in order to avoid muscle fatigue.

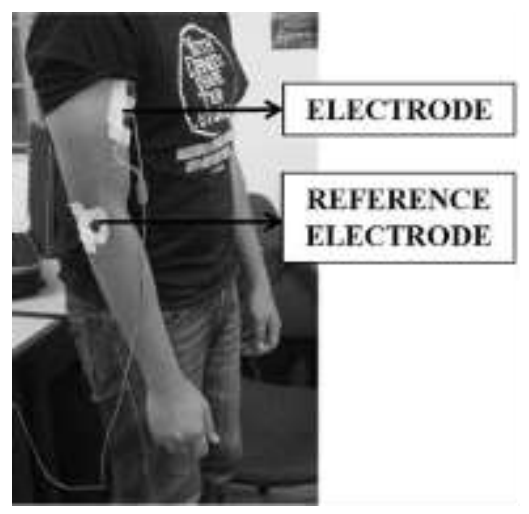

(a)

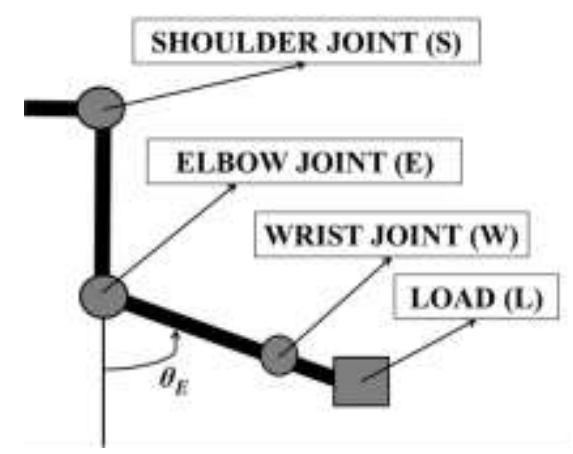

(b)

Figure 1. The experimental set-up: (a) electrodes placement; (b) position of hand during flexion.

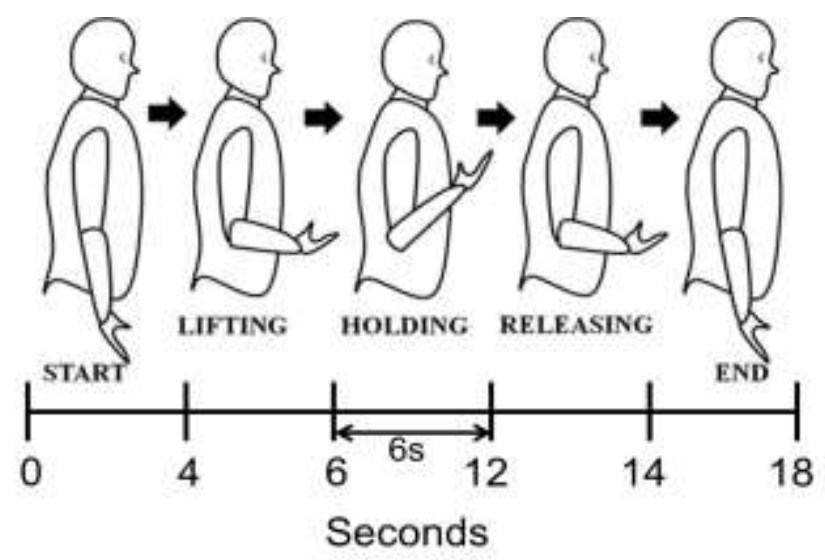

Figure 2. Movements of the subject.

\section{Subject}

A normal and healthy male subject participated in this study. The subject was selected according to the dominant hand; right-handed, and weight; above $60 \mathrm{~kg}$. The subject was a volunteer and had signed a consent form before the experiment was conducted. The consent form is a statement signed by the subject which acknowledges that the subject is a volunteer, understands and is aware of the consequences of the experiment that will be conducted. This is as a precaution should anything happen to the subject after the experiment.

\section{System Design}

This experiment uses a basic system for acquiring biosignals. Starting from the electrodes, data acquisition until signal processing was designed. The system design 
includes the sEMG self-adhesive silver-silver-chloride $(\mathrm{Ag} / \mathrm{AgCl})$ surface electrode used as a transducer to capture the EMG voltage signal. It was attached to the biceps brachii muscle on the right hand. $\mathrm{Ag} / \mathrm{AgCl}$ electrodes are chosen because their half-cell potential is closer to zero than other types such as silicon rubber electrodes. Three $\mathrm{Ag} / \mathrm{AgCl}$ electrodes were used in this experiment for the subject. Independent measurement can be obtained from the hand movement without moving the shoulder. The EMG signal captured from the surface electrodes was then digitized by the sEMG data acquisition system; TeleMyo 2400T G2 (Noraxon, USA). The sEMG data acquisition system is used to record EMG signals from the subject. The EMG signals were obtained by using the TeleMyo 2400T G2 Transmitter, which sends the signals by wireless transmission to the TeleMyo PC-Interface Receiver, which forwards the data via USB to the computer at a sampling rate of 1500 samples per second. A computer was used as a digital signal processing system, including digital rectification and features extraction.

\section{PROCESSING THE EMG SIGNAL}

\section{Rectification}

The recorded data of the raw EMG signals contain very important information and may be used as the first objective information and documentation of the muscle. The EMG signals consist of positive and negative phases that change about a baseline of zero voltage (isoelectric line). Rectification is important due to the fluctuation characteristic of the EMG signal about zero value. The implementation of the rectification is done via taking the absolute value of the sEMG signal, which is inverting the negative phases. In this study, the raw data of sEMG signals were rectified via the full-wave rectification method. This method used Eq. (1) to fully rectify the raw data of the sEMG signals.

$$
y_{i}=\left|x_{i}\right|
$$

\section{Time Domain Features Extraction}

Features in the time domain have been widely used in medical and engineering practices and researches. Time domain features are used in signal classification due to their performance in signal classification in low noise environments, their lower computational complexity, and easy and quick implementation. Furthermore, the features are calculated based on raw EMG time series. The time domain features assume the data as a stationary signal [14]. Moreover, much interference that is acquired through the recording because of their calculations is based on the EMG signal amplitude. Four time domain features have been proposed in this study through an extensive review of the literature. The information on statistical features extraction for the EMG signals was done in MATLAB R2011a. Four statistical features from the time domain are used in the evaluation, as follows:

- Integrated EMG (IEMG): IEMG $=\sum_{i=1}^{N} x_{i}$

- Mean absolute value (MAV): $M A V=\frac{1}{N} \sum_{i}^{N} x_{i}$ 
- Variance (VAR): $V A R=\frac{1}{N-1} \sum_{i}^{N} x_{i}^{2}$

- $\quad$ Root mean square (RMS): $R M S=\sqrt{\sum_{i}^{N} x_{i}^{2}}$

\section{RESULTS AND DISCUSSION}

\section{Characteristics of EMG Signal}

The EMG data in this study are measured during hand lifting without load and with dumbbell loads of $2 \mathrm{~kg}, 4 \mathrm{~kg}$ and $6 \mathrm{~kg}$. Figure 3 shows the raw EMG signals during the experiment. As can be observed in Figure 3, when the muscle contraction was maintained for a long period, the EMG signal amplitude decreased. This proves that the EMG signal is a non-stationary signal and the frequency of the signal changes over time. Furthermore, the relationship between changing in force during muscle contraction and increasing of load can be observed in Figure 3. The amplitudes of the EMG signals increase as the load increases. The amplitudes of the EMG signals for the $6 \mathrm{~kg}$ load as shown in Figure 3(d) are higher than for $4 \mathrm{~kg}$, while the amplitudes of the EMG signals for the $4 \mathrm{~kg}$ load as shown in Figure 3(c) are higher than the signals obtained when lifting the hand with a $2 \mathrm{~kg}$ load and no load. The amplitudes of EMG signals for no load have been selected as the reference amplitudes of the EMG signals in this experiment. Figure 3 shows that the EMG signals reach the maximum amplitude at the early stage of the recorded signal. This shows that the muscle contraction is high when the subject starts to lift the load, whereas the EMG signal amplitude decreased at the last stage of the recorded signal when the subject was lowering the load in order to release it [7].

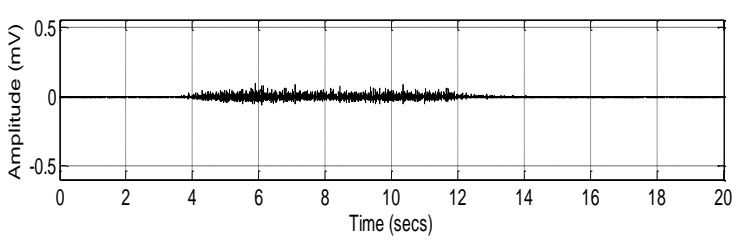

(a)

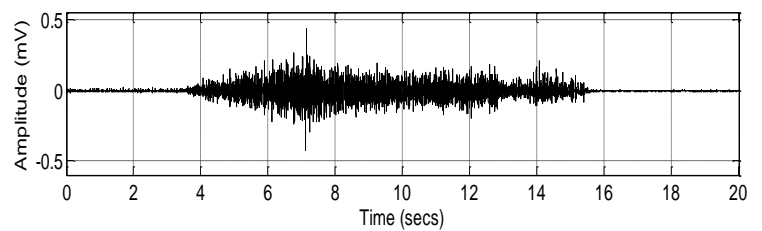

(c)

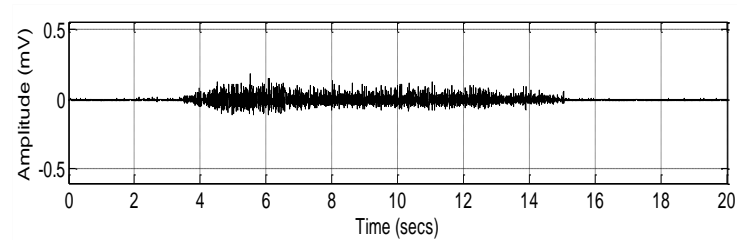

(b)

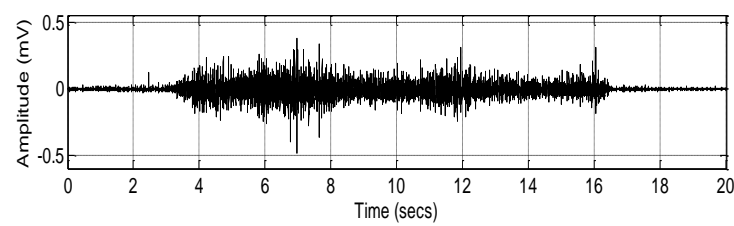

(d)

Figure 3. Raw EMG signals for: (a) no load; (b) $2 \mathrm{~kg}$; (c) $4 \mathrm{~kg}$; (d) $6 \mathrm{~kg}$ load.

\section{Assessing the EMG Features}

The EMG signals were analysed in the time domain to get the features. Overall, four time domain features were selected and analysed from the biceps brachii muscle for load lifting, namely IEMG, MAV, VAR and RMS. The average value from the load lifting with repeatability of 30 times was calculated. The purpose of this was to obtain 
the correlation between the EMG voltage amplitude and the loads. Table 2 shows the average value of time domain features for four different loads obtained from the EMG signals. As can be observed in Table 2, the values of the statistical features for the EMG signal differ for each load. The differences in the statistical feature values show that the EMG signals vary according to the changes of load. The statistical features increased when the load increased. The maximum amplitude of the EMG signals increased as the load increased. This is because more force is needed to lift the load when the load increases. From Figure 4, we can see the relationship between changes in load and the time domain features. Overall, the value of the features increases due to the increasing of the EMG signal amplitude according to the increasing weight of load. The results show that the value of the features differs between the different loads. This can be used to classify the EMG signal according to the time domain features and weight of loads $[15,16]$.

Table 2. Average value of time domain features for four different loads.

\begin{tabular}{rrrrrr}
\hline & \multicolumn{4}{c}{ Time domain features $(\mathrm{mV})$} \\
\cline { 3 - 6 } Load $(\mathrm{kg})$ & 0 & $\mathbf{1 4 9 . 2}$ & $7.6 \times 10^{-3}$ & \multicolumn{1}{c}{ VAR } & \multicolumn{1}{c}{ RMS } \\
\cline { 3 - 6 } & 2 & $\mathbf{3 0 2 . 2}$ & 0.014 & $4.969 \times 10^{-4}$ & 0.011 \\
& 4 & $\mathbf{4 6 2 . 5}$ & 0.024 & $1.57 \times 10^{-3}$ & 0.022 \\
& 6 & $\mathbf{6 5 2 . 2}$ & 0.030 & $3.28 \times 10^{-3}$ & 0.057 \\
& & & \\
R-squared $\left(\mathrm{R}^{2}\right)$ & $\mathbf{0 . 9 9 7}$ & 0.994 & 0.926 & 0.99
\end{tabular}
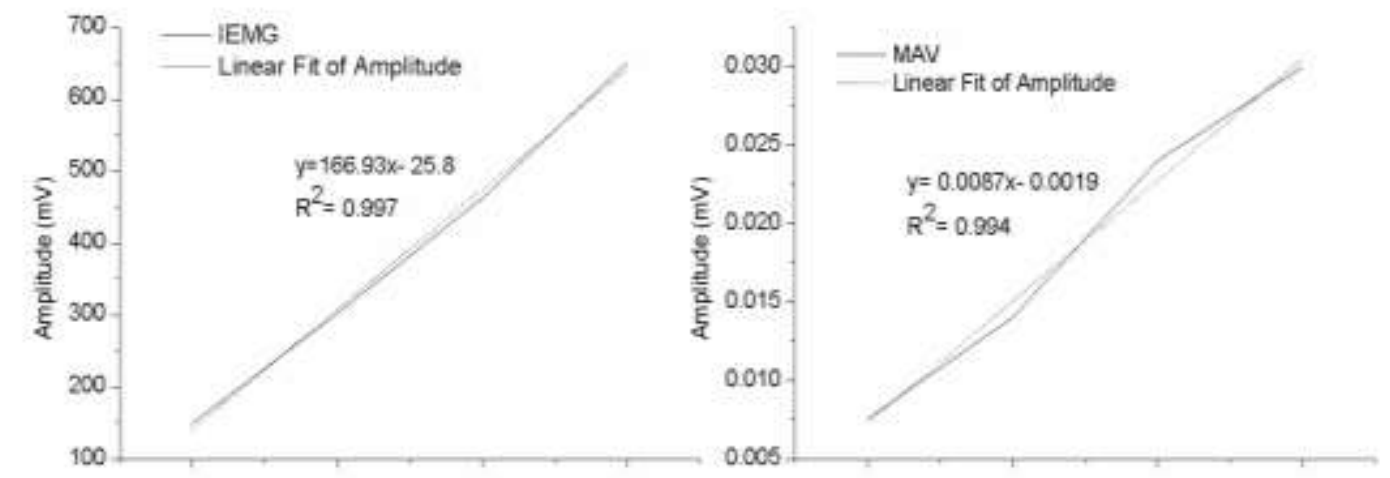

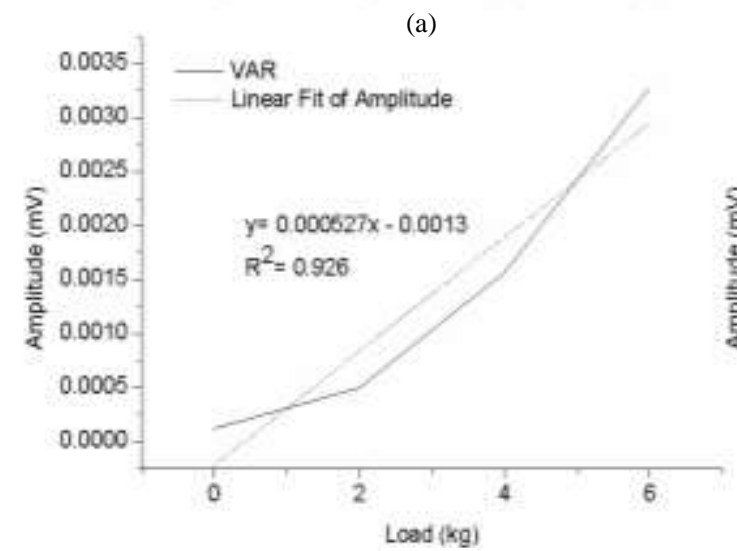

(c)

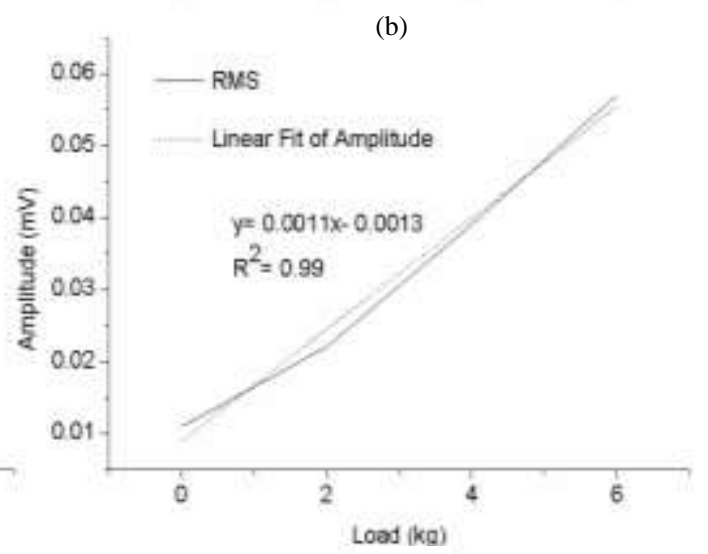

(d)

Figure 4. Relationship of time domain features with load: (a) IEMG; (b) MAV; (c) VAR; (d) RMS. 
It can be seen in Table 2 and Figure 4 that the correlations between loads and EMG voltage amplitude of all features are almost linear, with R-squared values near to 1.00 , which means that the correlation is good. Based on the results, IEMG shows the greatest linear correlation between loads and EMG voltage amplitude. The IEMG has an $\mathrm{R}$-squared value that is equal to 0.997 . The system will use IEMG as the reference feature to estimate the muscle's force. By using the full-wave rectification method, the average muscle force can be estimated using Eq. (8) [15].

$$
y=166.93 x-25.8
$$

By comparing the $\mathrm{y}$-axis and $\mathrm{x}$-axis,

$$
V=166.93 F-25.8
$$

Hence,

$$
F=0.006 V+0.155
$$

for muscle force with loads of $0 \mathrm{~kg}, 2 \mathrm{~kg}, 4 \mathrm{~kg}$ and $6 \mathrm{~kg}$.

In Eq. (8), $V$ is the EMG voltage and $F$ is the average muscle force. Note that this force should be multiplied with the gravity coefficient, $g$ [15]. This means that when $V$ is $200 \mathrm{mV}$, then $F$ is $0.1558 \mathrm{~g}$ Newton for the fully rectified method.

\section{CONCLUSIONS}

This study is targeted for researchers to look into the details of the features that can be extracted from the EMG signal during hand-lifting of four different loads. It is intended to allow for better interpretation of EMG signals analysis in the time domain. Four features have been extracted from the EMG signals: IEMG, MAV, VAR and RMS. Furthermore, the average muscle force condition can be estimated by the correlation between the EMG voltage amplitude with linear estimation with the full-wave rectification method. The R-squared value determined the correlation between the EMG voltage amplitude with the loads. IEMG was chosen as the reference feature for estimation of the muscle's force because of its R-squared value equal to 0.997. By referring to the IEMG, the linear equation obtained from the correlation was used for estimation of the muscle's force. These findings could be integrated to design a muscle force model based on the biceps.

\section{ACKNOWLEDGEMENTS}

The authors are so indebted and would like to express their thankfulness to the Robotics and Industrial Automation Research Group, Faculty of Electrical Engineering, Universiti Teknikal Malaysia Melaka (CERIA-UTeM) for their ideas and comments which helped improve this paper, and to the Ministry of Higher Education of Malaysia for funding this study under-VOT F00123 2012. 


\section{REFERENCES}

[1] Ahmed S, Ahmad S, Faruqe MO, Islam MR. EMG signal decomposition using wavelet transformation with respect to different wavelet and a comparative study. Proceedings of the 2nd International Conference on Interaction Sciences: Information Technology, Culture and Human. Seoul, Korea: ACM; 2009. p. $730-5$.

[2] Magaswaran K, Phuman Singh AS, Hassan MZ. A new method in the identification of noise and vibration characteristics of automotive disk brakes in the low frequency domain. International Journal of Automotive and Mechanical Engineering. 2014;9:1564-77.

[3] Merletti R, Parker PA. Electromyography: Physiology, Engineering, and NonInvasive Applications. New York: John Wiley and Sons Inc.; 2004.

[4] Erdemir A, McLean S, Herzog W, van den Bogert AJ. Model-based estimation of muscle forces exerted during movements. Clinical Biomechanics. 2007;22:131-54.

[5] Hug F. Can muscle coordination be precisely studied by surface electromyography? Journal of Electromyography and Kinesiology. 2011;21:112.

[6] Ahmad Z, Taha Z, Hassan HA, Hisham MA, Johari NH, Kadirgama K. Biomechanics measurements in archery. Journal of Mechanical Engineering and Sciences. 2014;6:762-71.

[7] Wan Daud WMB, Yahya AB, Chong SH, Sulaima MF, Sudirman R. Features extraction of electromyography signals in time domain on biceps Brachii muscle. International Journal of Modeling and Optimization. 2013;3:515-9.

[8] Parsaei H, Stashuk DW, Rasheed S, Farkas C, Hamilton-Wright A. Intramuscular EMG Signal Decomposition. Critical Reviews ${ }^{\mathrm{TM}}$ in Biomedical Engineering. 2010;38:435-65.

[9] Daud WMBW, Sudirman R. A wavelet approach on energy distribution of eye movement potential towards direction. Industrial Electronics \& Applications (ISIEA), 2010 IEEE Symposium on Industrial Electronics and Applications. Penang, Malaysia2010. p. 181-5.

[10] Abdul Rahman AG, Yee KS, Ismail Z, Kuan KK, Chao OZ, Tong CW, et al. Impact force identification using the modal transformation method in collocated and non-collocated cases. Journal of Mechanical Engineering and Sciences. 2014;6:968-74.

[11] Jamil N, Yusoff AR, Mansor MH. Literature review of electromagnetic actuator force generation for dynamic modal testing applications. Journal of Mechanical Engineering and Sciences. 2012;3:311-9.

[12] Hermens H, Merletti R, Freriks B. European activities on surface electromyography. Proceedings of the first general SENIAM (Surface EMG for Non Invasive Assessment of Muscles) workshop, September1996.

[13] Daud WMBW, Sudirman R. Time frequency analysis of electrooculograph (EOG) signal of eye movement potentials based on wavelet energy distribution. Fifth Asia Modelling Symposium. 2011, p. 81-6.

[14] Phinyomark A, Phukpattaranont P, Limsakul C. Feature reduction and selection for EMG signal classification. Expert Systems with Applications. 2012;39:742031. 
[15] Darmakusuma R, Prihatmanto AS, Indrayanto A, Mengko TL. Bicep brachii's force estimation using MAV method on assistive technology application. Instrumentation, Communications, Information Technology, and Biomedical Engineering (ICICI-BME), 2011 2nd International Conference on2011. p. 28892.

[16] Daud WMBW, Bukhari WM. Surface electromyography of eyes potential behaviour using wavelet transform analysis. Australian Journal of Basic and Applied Sciences. 2013;7:64-71. 